Haematology

Edited by Dr Drew Provan FRCP FRCPath,

Senior Lecturer in Haematology, The Royal London Hospital

\section{Haematological manifestations of}

\section{systemic disease}

Jerry L Spivak MD, Professor of Medicine and Oncology, Johns Hopkins University School of Medicine, Baltimore

Clin Med 2005;5:322-5

The major functions of the blood are to supply oxygen to the tissues, prevent microbial invasion and promote haemostasis. To ensure a sufficient number of cells for these functions, the bone marrow produces $10^{12}$ red cells, $10^{11}$ leucocytes and $10^{11}$ platelets daily to replace those lost through senescence or use. Because of this high rate of proliferative activity, the bone marrow is susceptible to disorders that:

- interfere with its anatomical integrity

- suppress the production of haematopoietic growth factors

- stimulate the production of inflammatory cytokines, or

- shorten the lifespan of circulating blood cells.

Indeed, systemic diseases frequently impairment or stimulation of blood cell production or their enhancement of blood cell destruction or sequestration. Thus, when confronted with an abnormality in haematopoiesis in an adult, it is first necessary to determine whether it is a primary blood disorder or secondary to an underlying systemic disease. This distinction is crucial for the appropriate management of the blood abnormality. When the haematological abnormality is secondary first become manifest through either their to a systemic disorder, correction of that disorder will alleviate the haematological abnormality, while haematinic therapy alone will not (Fig 1). There is, of course, no correlation between the severity of a blood abnormality and its underlying cause, which must be diligently sought if treatment is to be effective.

The evaluation of a blood disorder begins with the medical history, including prior blood counts and assessment of drug or toxin exposure, followed by a careful physical examination with attention to the presence of jaundice, telangiectasia, purpura, lymphadenopathy and splenomegaly. Careful examination of a blood smear is essential as is a reticulocyte count, which can distinguish decreased red cell production from increased red cell destruction unless both are present. A bone marrow biopsy is mandatory when there is pancytopenia or when bone marrow cannot be aspirated, in order to distinguish marrow aplasia from myelofibrosis or metastatic tumour in the bone marrow.

\section{Anaemia}

Anaemia is the most common abnormality associated with a systemic disease. The appropriate therapy for its correction will depend on the nature of the underlying disorder and the mechanism for the anaemia. Effective erythropoiesis represents the summation of four separate processes:

- the proliferative capacity of the erythroid progenitor cell pool

- the intensity of the stimulus for red cell production

- the adequate provision of nutrients, of which iron, folic acid and vitamin $\mathrm{B} 12$ are the most important, and

- red cell survival - this is ordinarily fixed in length, but can be shortened by premature red cell destruction or bleeding.

Table 1 lists the various causes of anaemia associated with systemic disorders. $^{1}$

\section{Red cell aplasia}

Red cell aplasia is a distinctive, but uncommon cause of anaemia associated with both solid tumours and haematological malignancies, as well as with

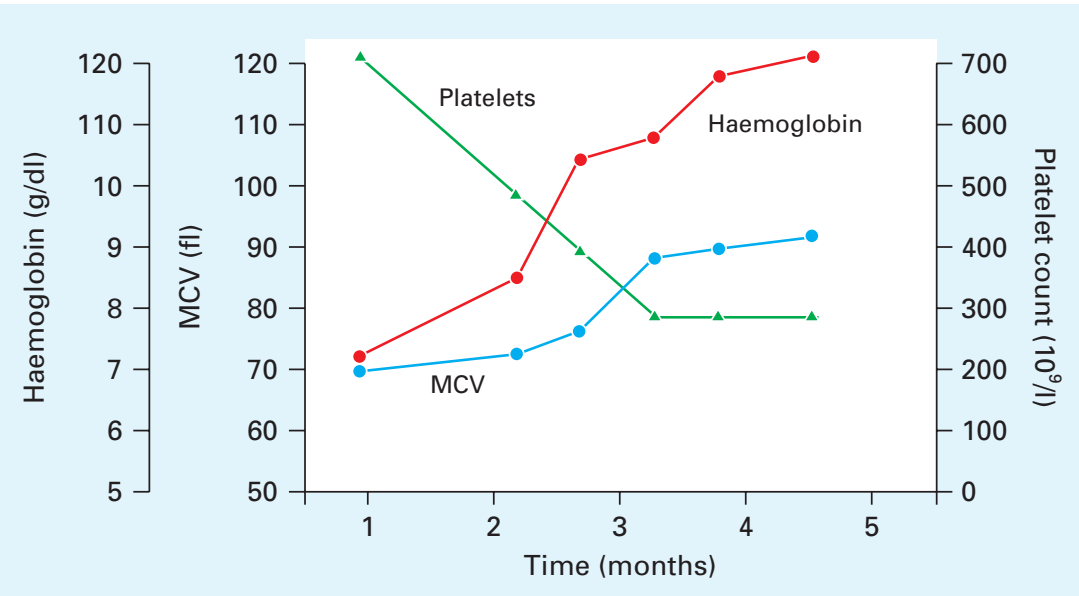

Fig 1. Haemoglobin, mean corpuscular volume (MCV) and platelet count before and after administration of prednisone to a 74-year-old woman with polymyalgia rheumatica (reproduced, with permission, from Ref 1 ). 
parvovirus B19 infection, autoimmune disorders and pregnancy. ${ }^{2}$ Certain drugs can also induce this type of anaemia, some of them possibly simply by creating an immunosuppressed state making the host susceptible to parvovirus B19 infection. A very low reticulocyte count $(<0.5 \%)$ or the absence of reticulocytes is the first clue to red cell aplasia; a bone marrow aspirate is required for absolute identification. Serum erythropoietin (EPO) is usually elevated in this disorder.

\section{Haemolysis}

Haemolysis as a consequence of a systemic disorder on a non-immune basis can be due to:

- infection with Clostridia, malaria, Bartonella or Babesia

- vascular damage or obstruction due to intravascular coagulation, vasculitis, malignant hypertension, eclampsia, disseminated cancer

- thrombotic microangiopathy, or

- Wilson's disease.

Infection can also initiate oxidative haemolysis in glucose-6-phosphate dehydrogenase-deficient erythrocytes.

On an immune basis, haemolysis can be due to:

- infection with malaria, syphilis (paroxysmal cold haemoglobinuria), Epstein-Barr virus and Mycoplasma (cold agglutinin disease), or

- warm autoantibody formation associated with a solid tumour, lymphoma or collagen vascular disease.

Examination of a blood smear is the most direct method for identifying the presence of a haemolytic process and usually its mechanism. The reticulocyte count is typically elevated when there is significant uncompensated haemolysis.

\section{Anaemia of chronic disease}

The type of anaemia designated 'anaemia of chronic disease' is a common complication of infectious, inflammatory and neoplastic disorders. Its recognition is important since its physiological correction depends on correction of the under- lying disorder causing the anaemia. Indeed, the presence of this form of anaemia is an important clue to the presence of an underlying systemic disorder.

The most common cause of anaemia associated with a systemic disorder is hypoproliferative, characterised by:

- mild to severe anaemia

- inappropriately low reticulocyte count and serum $\mathrm{EPO}^{3}$ for the degree of anaemia

- low serum iron and iron binding capacity

- low transferrin saturation

- adequate marrow storage iron, and

- normal or high serum ferritin in the absence of bleeding or haemolysis. ${ }^{4,5}$

Iron metabolism abnormalities and erythropoietin. The distinctive abnormalities of iron metabolism associated with the anaemia of chronic disease are widely considered to be a diagnostic feature. Although this is true, lack of available iron for red cell production is not the mechanism for the anaemia. Rather, it is lack of adequate EPO production, due in part to increased intracellular iron accumulation. ${ }^{6}$ In the anaemia of chronic dis- ease, inflammatory cytokines provoke the synthesis of hepcidin, a small antimicrobial peptide. ${ }^{7}$ Hepcidin blocks both iron absorption in the intestine and iron release by macrophages and other cells. Increased intracellular iron promotes degradation of hypoxia factor-1, necessary for upregulating EPO production. ${ }^{8}$ At the same time, inflammatory cytokines also impair EPO production by other mechanisms and the ability of erythroid progenitor cells to respond to EPO. ${ }^{9}$ Importantly, even if it is not possible to correct the underlying systemic disorder, administration of recombinant EPO often overcomes EPO resistance in this situation and alleviates the anaemia.

In this regard, it is important to emphasise that inflammation, infection and cancer are not the only systemic disorders that have a negative impact on EPO production. Diabetes mellitus and chronic hypertension impair renal endocrine function out of proportion to renal excretory function, and anaemia due to reduced EPO production can develop with only minimal serum creatinine elevation (Fig 2). ${ }^{3}$ It is also important to recognise that disorders impairing pituitary or thyroid function

\section{Table 1. Causes of anaemia associated with systemic diseases.}

$\begin{array}{ll}\text { Hypoproliferative anaemias } & \text { Inflammatory processes (collagen vascular diseases, IBD) } \\ \text { Infection } & \text { Cancer (solid tumours, lymphomas, multiple myeloma) } \\ & \text { Parenchymal renal disease } \\ & \text { Hypopituitarism } \\ & \text { Hypothyroidism } \\ & \text { Malabsorption syndromes (coeliac disease) } \\ & \text { Hyperviscosity (multiple myeloma) } \\ & \text { Haematological malignancies (myeloid and lymphoid) } \\ \text { Solid tumours (thymoma, lung, stomach, breast) } \\ \text { Immunological disorders (collagen vascular disorders) } \\ \text { Pregnancy } \\ \text { BMT cell aplasia } \\ \text { Infectious diseases (parvovirus B19, EBV, hepatitis A, B, C) } \\ \text { Infections (malaria, bartonellosis, babesiosis, Clostridia, EBV, } \\ \text { Mycoplasma) } \\ \text { Autoimmune disorders (collagen vascular diseases) } \\ \text { Cancer (solid tumours, lymphomas) } \\ \text { Wilson's disease } \\ \text { Pregnancy (HELLP syndrome, eclampsia) } \\ \text { Microangiopathic disorders (TTP, HUS, cancer, vasculitis, } \\ \text { malignant hypertension, DIC) }\end{array}$

$\mathrm{BMT}=$ bone marrow transplantation; $\mathrm{DIC}=$ disseminated intravascular coagulation; $\mathrm{EBV}=$ Epstein-Barr virus; HELLP = haemolysis, elevated liver enzymes and low platelet count syndrome; HUS = haemolytic uraemic syndrome; IBD = inflammatory bowel disease; TTP = thrombotic thrombocytopenic purpura. 


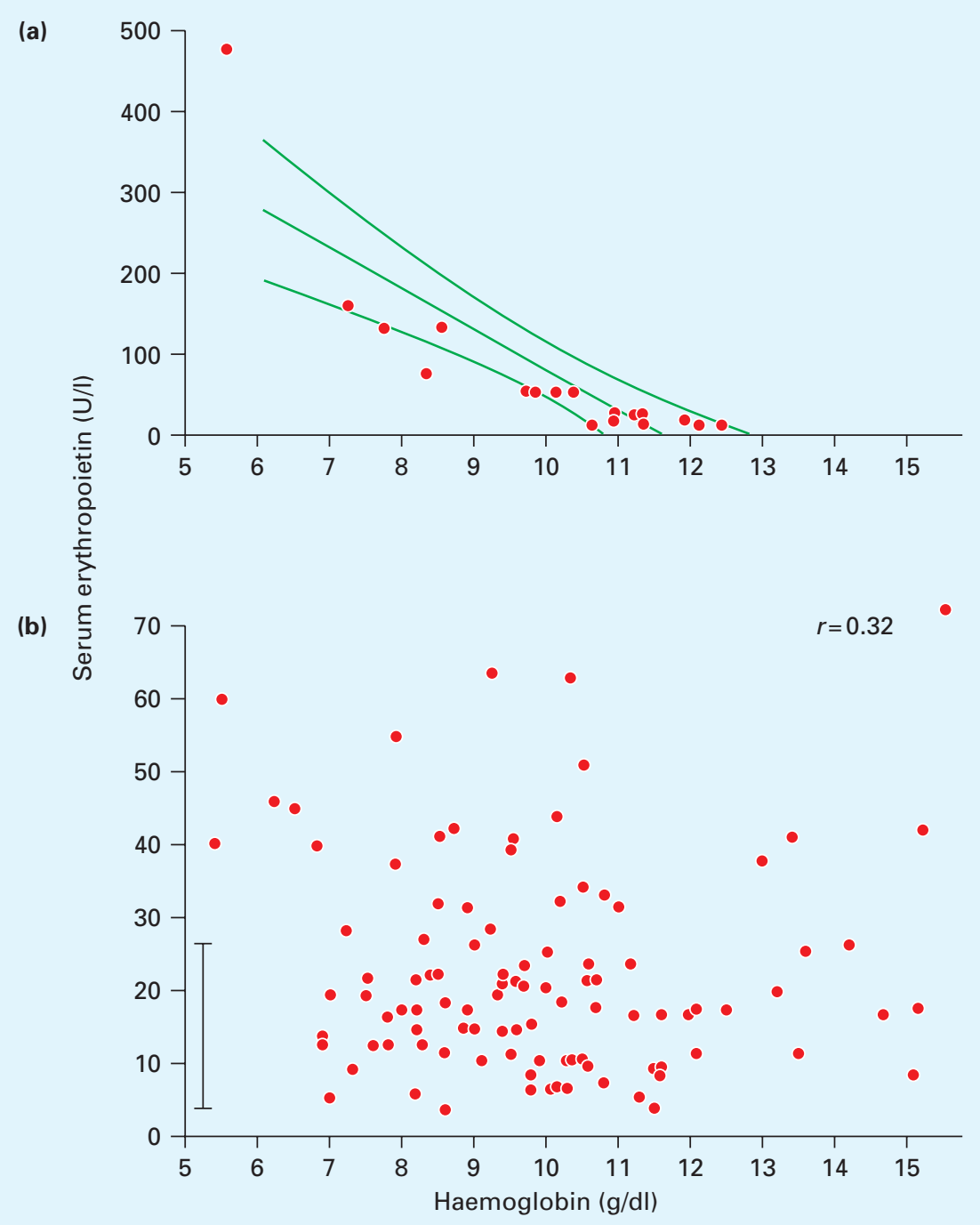

Fig 2. Relationship between serum erythropoietin (EPO) and haemoglobin (Hb) concentrations: (a) uncomplicated iron-deficiency anaemia (outer curves: $95 \%$ confidence intervals); (b) effect of a serum creatinine greater than $1.5 \mathrm{mg} \%$ $(>133 \mu \mathrm{m} / \mathrm{l})$ on the serum EPO-Hb relation (bar on EPO axis shows the normal range); $r=$ correlation coefficient (reproduced, with permission, from Ref 1).

\section{Key Points}

Systemic diseases often first become obvious through impairment of blood cell production

There is no correlation between the severity of the blood abnormality and the nature of the underlying cause

Anaemia is the most common abnormality associated with systemic disease

Recognition of the anaemia of chronic disorders is important since correction of the anaemia is dependent upon correction of the underlying systemic disease

Small molecules, such as hepcidin, appear to play a key role in the development of the anaemia of chronic disease through interference with iron absorption from the gut, as well as impairing iron release from macrophages

KEY WORDS: anaemia, anaemia of chronic disease, coagulation, leukocytes, platelets will lead to a reduction in basal metabolic rate, reducing tissue oxygen requirements and consequently EPO production. Intestinal disorders such as coeliac disease lead to anaemia due to malabsorption of iron and other key nutrients.

\section{Leucocytes}

Impaired leucocyte production or increased leucocyte destruction due to a systemic disorder is less common than abnormalities of red cell production and can be due to autoantibodies, cytotoxic lymphocytes (Felty's syndrome), infection, myelofibrosis, hyperthyroidism, splenic sequestration or a protein-losing enteropathy.

\section{Platelets}

Impaired platelet production or increased platelet destruction can be due to disseminated intravascular coagulation (DIC), red cell transfusion, thrombotic thrombocytopenic purpura (TTP), sepsis, autoantibodies, vasculitis, cancer, infection, inflammation or splenic sequestration.

\section{Pancytopenia}

Pancytopenia is an uncommon blood complication of a systemic illness and can be due to marrow aplasia secondary to a thymoma or an autoimmune disorder, infection causing marrow necrosis, splenic sequestration, pregnancy, hepatitis or the haemophagocytic syndrome.

\section{Excess blood cell production}

Overproduction of red cells, white cells or platelets is the least common consequence of a systemic disorder. However, tumours such as hepatomas or hypernephromas have been associated with an increase in one or more of the formed elements of the blood, while inflammatory or infectious disorders can be associated with a substantial leucocytosis or thrombocytosis. Fortunately, elevations of either as a reactive phenomenon do not require therapy per se. Erythrocytosis, however, is a special situation 
in which the distinction between a primary cause for the erythrocytosis and a secondary cause can initially be difficult, but phlebotomy can control the process while the diagnostic evaluation is continuing.

\section{Disorders of coagulation}

Systemic disorders can cause disturbances in blood coagulation not only by their quantitative effects on platelet production and survival but also by altering the balance between procoagulant and antithrombotic proteins in the blood. Suppression of platelet production or an increase in platelet destruction is probably the commonest mechanism by which systemic disorders disturb blood coagulation. Cancer, pregnancy, infection, collagen vascular and autoimmune disorders can create a hypercoagulable state by:

- inappropriate release of procoagulant proteins

- stimulating the production of procoagulant, antiphospholipid antibodies

- damaging endothelial surfaces, or

- causing hyperviscosity.

In its most extreme form - DIC - both thrombotic and haemorrhagic manifestations may be present due to the consumption of clotting factors and platelets and the production of fibrin degradation products that act as an anticoagulant. As with the other haematological complications of systemic diseases, correction of the underlying disorder is the most effective remedy for correcting the coagulopathy. Erythrocytosis should be considered a hypercoagulable state, but usually thrombocytosis not due to a haematological malignancy does not predispose to thrombosis or bleeding. ${ }^{10}$

\section{References}

1 Spivak JL. The blood in systemic disorders. Review. Lancet 2000;355:1707-12.

2 Fisch P, Handgretinger R, Schaefer HE. Pure red cell aplasia. Review. Br J Haematol 2000; 111:1010-22.

3 Spivak JL. The clinical physiology of erythropoietin. Review. Semin Hematol 1993; 30(4 Suppl 6):2-11.
4 Cartwright GE. The anemia of chronic disorders. Review. Semin Hematol 1966;3: 351-75.

5 Cash JM, Sears DA. The anemia of chronic disease: spectrum of associated diseases in a series of unselected hospitalized patients. Am J Med 1989;87:638-44.

6 Spivak JL. Iron and the anemia of chronic disease. Review. Oncology (Huntingt) 2002; 16(9 Suppl 10):25-33.

7 Nicolas G, Chauvet C, Viatte L, Danan JL et al. The gene encoding the iron regulatory peptide hepcidin is regulated by anemia, hypoxia, and inflammation. J Clin Invest 2002;110:1037-44.

8 Harris AL. Hypoxia - a key regulatory factor in tumour growth. Review. Nat Rev Cancer 2002;2:38-47.

9 Means RT Jr, Krantz SB, Luna J, Marsters SA, Ashkenazi A. Inhibition of murine erythroid colony formation in vitro by interferon gamma and correction by interferon receptor immunoadhesin. Blood 1994;83: 911-5.

10 Buss DH, Stuart JJ, Lipscomb GE. The incidence of thrombotic and hemorrhagic disorders in association with extreme thrombocytosis: an analysis of 129 cases. Am J Hematol 1985;20:365-72. 